\title{
Robust Stability Analysis for Linear Systems with Uncertain Fast-Varying Time Delay arising from Networked Control Systems
}

\author{
Juan-Eduardo Velázquez-Velázquez, Rosalba Galván-Guerra \\ Instituto Politécnico Nacional, UPIIH, Hidalgo, \\ Mexico \\ \{jvelazquezv, rgalvang\}@ipn.mx
}

\begin{abstract}
This contribution is concerned with the robust stability analysis for linear systems with time-varying delay arising from networked control systems. The time-varying delay is supposed to be bounded and fast-varying. By constructing a new complete Lyapunov-Krasovskii functional, delay-dependent robust stability conditions are derived in terms of Linear Matrix Inequalities. The derivative of the new complete nominal Lyapunov-Krasovskii functional along the trajectories depends on the complete state and the state derivative.
\end{abstract}

Keywords: Robust stability, time delay systems, Lyapunov-Krasovskii functional, networked control systems.

\section{Introduction}

Conventional control systems are composed of interconnected controllers, sensors and actuators following a point-to-point architecture. As an alternative, the use of a multipurpose shared network to connect spatially distributed elements results in flexible architectures and generally reduces installation and maintenance costs [7]. These kind of systems are known as Networked Control Systems (NCSs). The relevance of NCSs is due to their broad range of application as mobile sensor networks [14], remote surgery [12], automated highway systems and unmanned aerial vehicles $[15,16]$. In contrast to using several dedicated independent connections, the use of a shared network introduces new challenges as the presence of time-varying network-induced delays, aperiodic sampling or packet dropouts. Since an implementable model usually needs to possess some stability properties, in this work we are interested in the robust stability analysis for a class of networked control systems (NCSs) where the control loops are closed through a real-time network forming a delay control system.

The dynamical systems with time delays commonly known as Time-Delay Systems (TDSs) is a continuous field of interest and development. Principally, because time-delay is one of the main causes of instability of systems. Nowadays, with the increasing expectations of dynamics performance the robust stability analysis of TDSs is of practical significance. Time-delay robustness is often studied for situations in which the delay is uncertain but remains constant throughout time. While much research has 
been done and stability criteria have been derived for systems with uncertain constant time-delays, the recent research has been focused in the case where the time delay is time varying. The significance of such research effort is tied to the interest in designing control algorithms for large-scale networked systems.

Most of the results devoted to the stability of TDSs with uncertain delays consider as assumption the stability of the system free of delays, and next, in the time domain, use appropriate Lyapunov-Razumikhin functions or Lyapunov-Krasovkii Functionals (LKFs) combined with Linear Matrix Inequalities (LMIs) to derive some upper bounds on the delay values $\mu$. This type of delays is considered as uncertain small delays with zero nominal values and perturbations from $[0, \mu][17,3,13]$. There are cases where the previous assumption, the stability of system free of delays fails, but it may be stable for some non-zero delay. This type of delays is called non-small delays [1] and their stability analysis cannot be performed by using simple LKFs [5]. In order to treat effectively the fast-varying delay commonly the proposed LKFs involve terms such as $\int_{-h}^{0} \int_{t+\theta}^{t} \dot{x}(s) W \dot{x}(s) d s d \theta$ and then the derivative along trajectories of the involved functionals present quadratic derivative terms. This is the reason why in [2] negative quadratic terms, depending on $\dot{x}(t)$ were inserted into $\dot{v}_{n}$. There, the compressive techniques for stability analysis of uncertain time-delay systems: free weighting matrices and application of Jensens inequality [5] were extended to the case of complete LKF. The derivative of the complete LKF in [2] depends on the present state only.

The complete type LKF introduced in [11] are constructed on the base of the solution of a linear matrix differential-difference equation on a finite time interval which satisfies additional symmetry and boundary conditions. This solution is called a delay Lyapunov matrix and it inherits properties of the classical quadratic Lyapunov functions for ordinary delay free differential equations. These LKFs prove to be useful in the robustness analysis of time delay systems [11]. In [10], the complete LKF was constructed for the uncertain system, which did not explicitly depend on the bounds of the uncertainties. As a result, the stability conditions were rather complicated, and induced some conservatism. In [4] a 'complete' LKF, depending on two delay Lyapunov like matrices, is constructed whose derivative along the trajectories depend on the state and its derivative which allows a less conservative treatment of the delay perturbation.

In this contribution, inspired in [11], [4], and [2], we enlarge the class of functionals by adding the functionals whose time derivative includes a given derivative term and functionals which correspond to the perturbed system. It is worth to mention, that the construction of the new proposed functional depends only on the delay Lyapunov matrix. The new complete LKF is used to obtain delay-dependent robust stability conditions for TDSs arising from NCSs. The paper is organized as follows: the problem statement is presented in Section II. Because of their key role in obtaining the sufficient robust stability conditions, some basic results concerning the quadratic LKF of complete type are recalled in Section III and also a new LKF is constructed. In Section IV we show how the new functional of complete type is used for the robust stability analysis of the system with uncertain time-varying delay. The main result of the contribution is illustrated with an example. In Section V some concluding remarks end the contribution. 


\section{Problem Statement}

In this work we consider a simple NCS setup shown in Fig. 1 with "initial" and "causal" delays $\tau_{a}, \tau_{b}$. By considering lumped delays a simple delayed model of the linear NCS is (see [18] for more details)

$$
\dot{x}(t)=A x(t)+B u\left(t-\tau_{a}-\tau_{b}\right), \quad u(t)=K x(t),
$$

where $x(t) \in \mathbb{R}^{n}$ is the state variable, $u(t) \in \mathbb{R}^{m}$ is a control input vector; $A \in \mathbb{R}^{n \times n}$, and $B \in \mathbb{R}^{n \times m}$ are known real constant matrices. The corresponding closed-loop system has the following TDS with time-varying state form:

$$
\dot{x}(t)=A_{0} x(t)+A_{1} x(t-\tau(t)), \quad x(t)=\varphi(t), \quad t \in[-\bar{h}, 0],
$$

where $x(t) \in \mathbb{R}^{n}$ is the system state at time $t, t \geq 0 ; A_{0}=A, A_{1}=B K$ are known constant real matrices with appropriate dimensions, $\varphi$ is a continuously differentiable vectorvalued initial function and $\bar{h}$ is an upper-bound on the time delay $\tau(\cdot)$. The state $x_{t}$ is defined by $x_{t}(\theta)=x(t+\theta)$ for $\theta \in[-\bar{h}, 0]$. The uncertain delay $\tau(t)$ is supposed to

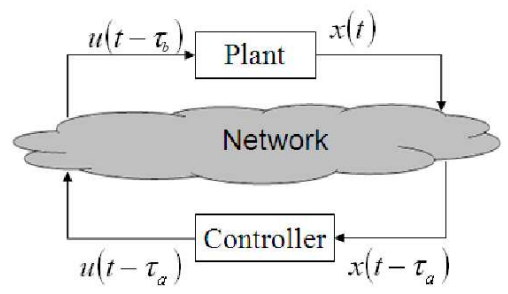

Fig. 1. A simple NCS with delays.

have the form $\tau(t)=h+\eta(t)$, where $h>0$ is a nominal constant value and $\eta(\cdot)$ is a time-varying perturbation. We assume that $\eta(t)$ is a sign-varying piecewise continuous function satisfying

$$
|\eta(t)| \leq \mu \leq h
$$

with known upper-bound $\mu$, i.e. $\tau \in[h-\mu, h+\mu]$. We consider the following assumption:

A1 Given the constant nominal value of the delay $h>0$, the nominal TDS

$$
\dot{x}(t)=A_{0} x(t)+A_{1} x(t-h),
$$

is exponentially stable.

Problem 1. Determine the value of $\mu$ for which the uncertain TDS with time-varying state (2) remains stable provided that the nominal system (4) is exponentially stable. 


\section{Preliminaries}

As suggested in [4] we consider the following form of LKF:

$$
v=v_{n}+v_{a},
$$

where $v_{n}$ is a nominal complete LKF which corresponds to the necessary and sufficient conditions for stability of the nominal system (4) and $v_{a}$ consists of additional terms and depends on $\mu$ and $v_{a} \rightarrow 0$ for $\mu \rightarrow 0$. Therefore, for $\mu \rightarrow 0 v \rightarrow v_{n}$. The latter will guarantee that if the conditions for the stability of the nominal system are feasible, then the stability conditions for the perturbed system will be feasible for small enough $\mu$.

The LKFs of complete type are such that their derivative along the solutions of the system is more substantial than a quadratic form of the state, allowing to prove that they admit a quadratic lower bound [11]. Under assumption A1, given a continuous initial function $\varphi(\theta)$ in $\theta \in[-h, 0]$, there exists a nominal complete LKF [11] of the form

$$
\begin{aligned}
& v_{0}\left(x_{t}, W\right)=x^{T}(t) U(0) x(t)+2 x^{T}(t) \int_{-h}^{0} U(-h-\theta) A_{1} x(t+\theta) d \theta+\int_{-h}^{0} \int_{-h}^{0} x^{T}\left(t+\theta_{2}\right) A_{1}^{T} \\
& \times U\left(\theta_{2}-\theta_{1}\right) A_{1} x\left(t+\theta_{1}\right) d \theta_{1} d \theta_{2}+\int_{-h}^{0} x^{T}(t+\theta)\left[W_{1}+\left(h_{1}+\theta\right) W_{2}\right] x(t+\theta) d \theta,
\end{aligned}
$$

whose time derivative along nominal system (4) is $\left.\frac{d}{d t} v_{0}\left(x_{t}\right)\right|_{(4)}=-w_{0}\left(x_{t}\right)$, where

$$
w_{0}\left(x_{t}\right)=x^{T}(t) W_{0} x(t)+x^{T}(t-h) W_{1} x(t-h)+\int_{-h}^{0} x^{T}(t+\theta) W_{2} x(t+\theta) d \theta
$$

and $W=W_{0}+W_{1}+h W_{2}$.

Observe that functional (6) is defined by the matrix valued function $U(\cdot)$ known as delay Lyapunov matrix. This matrix is as important for the functionals, as the classical Lyapunov matrix is for the quadratic Lyapunov functions in the case of delay free systems. Also note that the derivative of the LKF (7) depends on the complete state of the system.

Definition 1. [9] The $n \times n$ matrix $U(s)$ is a delay Lyapunov matrix of system (4) associated with a symmetric matrix $W$ if it satisfies the properties:

$$
\begin{gathered}
U^{\prime}(s)=U(s) A_{0}+U(s-h) A_{1}, \quad s \geq 0, \\
U(-s)=U^{T}(s), \quad s \geq 0, \\
-W=U(0) A_{0}+A_{0}^{T} U(0)+U(-h) A_{1}+A_{1}^{T} U(h) .
\end{gathered}
$$

The existence and uniqueness issues of the delay Lyapunov matrix are investigated in $[9,8]$. With the choice of $W=W_{0}+W_{1}+h W_{2}$ we can obtain a delay Lyapunov matrix $U(\cdot)$ as the solution of Eq. (8) satisfying conditions (9) and (10), which can be used for 
the construction of the LKF (6). The functional (6) is said to be of complete type if matrices $W_{i}, i=0,1,2$, are positive definite.

In order to treat effectively the fast-varying delay it is necessary that the derivative along trajectories of the involved functionals present quadratic derivative terms. Then in the following subsection we construct a new LKF whose derivative along trajectories of the nominal system (4) presents quadratic derivative terms. The construction of the new LKF unlike the one presented in [4] depends only in the delay Lyapunov matrix, see Definition 1.

\subsection{A New LKF for The Nominal TDS}

A broader class of functionals can be useful for achieving less conservative delaydependent stability conditions for uncertain non-small delays. We look now for functionals of complete type that along with the nominal system (4) satisfy

$$
\dot{v}_{n}\left(x_{t}\right)=-w_{n}\left(x_{t}\right)
$$

with $w_{n}\left(x_{t}\right)=w_{0}\left(x_{t}\right)+\bar{w}_{n}\left(x_{t}\right)$, where $w_{0}(\cdot)$ is defined in (7) and

$$
\bar{w}_{n}\left(x_{t}\right)=\dot{x}^{T}(t) Z \dot{x}(t)+x^{T}(t) Z A_{0} \dot{x}(t)+\dot{x}^{T}(t) A_{0}^{T} Z x(t) .
$$

We assume that the matrix $Z$ is symmetric and such that

$$
\left[\begin{array}{cc}
W_{0}+A_{0}^{T} Z A_{0}+Z A_{0} A_{0}+A_{0}^{T} A_{0}^{T} Z & A_{0}^{T} Z A_{1}+Z A_{0} A_{1} \\
A_{1}^{T} Z A_{0}+A_{1}^{T} A_{0}^{T} Z & W_{1}+A_{1}^{T} Z A_{1}
\end{array}\right]>0 .
$$

Substituting into $\bar{w}_{n}\left(x_{t}\right), A_{1} x(t-h)$ by $\dot{x}(t)-A_{0} x(t)$ and considering the nominal system (4), we find that

$$
\bar{w}_{n}\left(x_{t}\right)=-x^{T}(t) A_{0}^{T} Z A_{0} x(t)+x^{T}(t-h) A_{1}^{T} Z A_{1} x(t-h)+\frac{d}{d t} x^{T}(t)\left(A_{0}^{T} Z+Z A_{0}\right) x(t) .
$$

Next, by assumption A1 the integration from 0 to $\infty$ of expression (11) gives

$$
\begin{gathered}
v_{n}\left(x_{t}\right)=-x^{T}(t)\left[A_{0}^{T} Z+Z A_{0}\right] x(t)+\int_{0}^{\infty} x^{T}(t)\left[W_{0}-A_{0}^{T} Z A_{0}\right] x(t) d t \\
+\int_{0}^{\infty} x^{T}(t-h)\left[W_{1}+A_{1}^{T} Z A_{1}\right] x(t-h) d t+\int_{0}^{\infty} \int_{-h}^{0} x^{T}(t+\theta) W_{2} x(t+\theta) d \theta d t .
\end{gathered}
$$

If we define

$$
W=W_{0}+W_{1}+h W_{2}-A_{0}^{T} Z A_{0}+A_{1}^{T} Z A_{1}
$$


we can obtain a LKF constructed with delay Lyapunov matrix $U(\cdot)$ associated with matrix (13). The functional $v_{n}\left(x_{t}, W\right)$ is of the form

$$
\begin{aligned}
v_{n}\left(x_{t}, W\right)= & x^{T}(t)\left[U(0)-A_{0}^{T} Z-Z A_{0}\right] x(t)+2 x^{T}(t) \int_{-h}^{0} U(-h-\theta) A_{1} x(t+\theta) d \theta \\
& +\int_{-h-h}^{0} \int^{0} x^{T}\left(t+\theta_{2}\right) A_{1}^{T} U\left(\theta_{2}-\theta_{1}\right) A_{1} x\left(t+\theta_{1}\right) d \theta_{1} d \theta_{2} \\
& +\int_{-h}^{0} x^{T}(t+\theta)\left[W_{1}+A_{1}^{T} Z A_{1}+(h+\theta) W_{2}\right] x(t+\theta) d \theta .
\end{aligned}
$$

Observe that the new LKF (14) depends only in the delay Lyapunov matrix, see Definition 1. Also note that it preserves the structure of the nominal functional (6).

\section{Robust Stability Analysis}

In this section, for the robust stability analysis of the perturbed system (2) the nominal system (4) is assumed to be stable. We derive the values $\mu$ for which system (2) remains asymptotically stable for all perturbation values $\eta(t)$ satisfying (3). To obtain such bounds following [10], for $t \geq 0$, we represent the perturbed system in the form

$$
\dot{x}(t)=A_{0} x(t)+A_{1} x(t-h)-A_{1} \int_{t-h-\eta(t)}^{t-h} \dot{x}(s) d s .
$$

We consider the $\operatorname{LKF}(5), v=v_{n}+v_{a}$, where $v_{n}$ is the new complete LKF (14) and $v_{a}$ is the LKF which corresponds to the uncertainty

$$
\begin{aligned}
v_{a}\left(x_{t}\right)= & \mu \int_{t-h}^{t} x^{T}(s) S x(s) d s+\int_{-\mu}^{\mu} \int_{t+\theta-h}^{t} \dot{x}^{T}(s) R \dot{x}(s) d s d \theta \\
& +\mu r \int_{-h t+\theta}^{0} \int^{t} x^{T}(s) A_{1}^{T} U(\theta+h) U^{T}(\theta+h) A_{1} x(s) d s d \theta,
\end{aligned}
$$

with $S, R$, are $n \times n$ positive matrices and $r$ a positive scalar. Observe that $v \rightarrow v_{n}$ when $\mu \rightarrow 0$.

Then, by using only the delay Lyapunov matrix properties (8)-(10), the time derivative of (5) along the trajectories of the perturbed system (15) is obtained. First, for the new LKF (14) by straightforward computation we obtain that $\dot{v}_{n}\left(x_{t}, W\right)=-w_{n}\left(x_{t}\right)+\Delta$, where

$$
\Delta=-2\left[A_{t} \int_{t-h-\eta(t)}^{t-h} \dot{x}(s) d s\right]^{T}\left[\left[U(0)-A_{0}^{T} Z-Z A_{0}\right] x(t)+\int_{-h}^{0} U^{T}(h+\theta) A_{1} x(t+\theta) d \theta\right] .
$$


Considering the perturbed system $(15), \dot{v}_{n}(\cdot)$ is rewritten as

$$
\begin{gathered}
\dot{v}_{n}\left(x_{t}\right)=-w_{0}\left(x_{t}\right)-\dot{x}^{T}(t) Z \dot{x}(t)-2 x^{T}(t) Z A_{0} \dot{x}(t)-2\left[A_{1} \int_{t-h-\eta(t)}^{t-h} \dot{x}(s) d s\right]^{T} \\
\times\left[\left[U(0)-Z A_{0}\right] x(t)+Z \dot{x}(t)+\int_{-h}^{0} U^{T}(h+\theta) A_{1} x(t+\theta) d \theta+\frac{1}{2} Z A_{1} \int_{t-h-\eta(t)}^{t-h} \dot{x}(s) d s\right] .
\end{gathered}
$$

Next, we calculate the derivative of the functional $v_{a}\left(x_{t}\right)$ along the trajectories of the perturbed system (15):

$$
\begin{aligned}
\dot{v}_{a}\left(x_{t}\right)= & \mu x^{T}(t) S x(t)-\mu x^{T}(t-h) S x(t-h)+2 \mu \dot{x}^{T}(t) R \dot{x}(t)-\int_{t-h-\mu}^{t-h+\mu} \dot{x}^{T}(s) R \dot{x}(s) d s \\
& +\mu r x^{T}(t) A_{1}^{T} \mathscr{U} A_{1} x(t)-\mu r \int_{-h}^{0} x^{T}(t+\theta) A_{1}^{T} U(\theta+h) U^{T}(\theta+h) A_{1} x(t+\theta) d \theta,
\end{aligned}
$$

where

$$
\mathscr{U}=\int_{-h}^{0} U(\theta+h) U^{T}(\theta+h) d \theta
$$

Applying the Jensen integral inequality [5], we obtain the following estimations:

$$
\begin{gathered}
\int_{t-h-\mu}^{t-h+\mu} \dot{x}^{T}(s) R \dot{x}(s) d s \geq \int_{t-h-\eta(t)}^{t-h} \dot{x}^{T}(s) R \dot{x}(s) d s \geq \frac{1}{\mu} \int_{t-h-\eta(t)}^{t-h} \dot{x}^{T}(s) d s R \int_{t-h-\eta(t)}^{t-h} \dot{x}(s) d s \\
\int_{-h}^{0} x^{T}(t+\theta) A_{1}^{T} U(\theta+h) U^{T}(\theta+h) A_{1} x(t+\theta) d \theta \\
\geq \frac{1}{h} \int_{-h}^{0} x^{T}(t+\theta) A_{1}^{T} U(\theta+h) d \theta \int_{-h}^{0} U^{T}(\theta+h) A_{1} x(t+\theta) d \theta
\end{gathered}
$$

It is a fact that the use of free-weighting matrices reduce more the conservatism of the delay-dependent stability conditions [6]. Then we insert into $\dot{v}\left(x_{t}\right)$ negative quadratic terms, depending on $\dot{x}(t)$ by adding the following zero term:

$$
\delta=2\left[-\dot{x}(t)+A_{0} x(t)+A_{1} x(t-h)-A_{1} \int_{t-h-\eta(t)}^{t-h} \dot{x}(s) d s\right]^{T}\left[N_{1} x(t)+N_{2} \dot{x}(t)\right]=0,
$$

where $N_{1}$ and $N_{2}$ are free $n \times n$ matrices. 
Considering the previous estimations and inserting the zero term $\delta$, the time derivative of the LKF (5) admits the following estimation:

$$
\dot{v}\left(x_{t}\right) \leq \zeta^{T} \Phi \zeta
$$

where

$$
\zeta^{T}=\left[x^{T}(t) \dot{x}^{T}(t) \frac{1}{\mu} \int_{t-h-\eta(t)}^{t-h} \dot{x}^{T}(s) d s \int_{-h}^{0} x^{T}(t+\theta) A_{1}^{T} U(\theta+h) d \theta x^{T}(t-h)\right]
$$

and

$$
\Phi=\left[\begin{array}{ccccc}
\phi_{11} & \phi_{12} & \phi_{13} & \phi_{14} & \phi_{15} \\
* & \phi_{22} & \phi_{23} & \phi_{24} & \phi_{25} \\
* & * & \phi_{33} & \phi_{34} & \phi_{35} \\
* & * & * & \phi_{44} & \phi_{45} \\
* & * & * & * & \phi_{55}
\end{array}\right]
$$

here $\phi_{11}=-W_{0}+A_{0}^{T} N_{1}+N_{1}^{T} A_{0}+\mu r A_{1}^{T} \mathscr{U} A_{1}+\mu S, \phi_{12}=A_{0}^{T} N_{2}-N_{1}^{T}-Z A_{0}, \phi_{13}=$ $-\mu\left[U(0)-Z A_{0}+N_{1}\right]^{T} A_{1}, \phi_{14}=0, \phi_{15}=N_{1}^{T} A_{1}, \phi_{22}=-Z-N_{2}-N_{2}^{T}+2 \mu R, \phi_{23}=$ $-\mu\left[Z+N_{2}\right]^{T} A_{1}, \phi_{24}=0, \phi_{25}=N_{2}^{T} A_{1}, \phi_{33}=-\mu R-\mu^{2} A_{1}^{T} Z A_{1}, \phi_{34}=-\mu A_{1}^{T}, \phi_{35}=0$, $\phi_{44}=-\frac{\mu r}{h} I_{n \times n}, \phi_{45}=0, \phi_{55}=-W_{1}-\mu S$, hereafter $*$ stands for symmetric elements of the symmetric matrix. We have proved the following result which solves Problem 1.

Theorem 1. Assume that the nominal TDS (4) satisfies A1. Given $n \times n$-positive definite matrices $W_{i}, i=0,1,2$. Let $Z$ be defined by (12), $U(\cdot)$ be defined by (8)-(10) with $W$ satisfying (13), and $\mathscr{U}$ be defined by (17). Then, the uncertain system (15) is asymptotically stable for a continuous time-varying function $|\eta(t)|<\mu$ if there exist $n \times n$-matrices $N_{1}$, $N_{2}$, and $n \times n$-positive definite matrices $R$ and $S$, and a positive scalar $r$ such that the LMI: $\Phi<0$ holds, where $\Phi$ is that of (18).

\subsection{A Benchmark Comparison}

In this subsection, the combination of functionals with prescribed time derivative including quadratic derivative terms, the functionals corresponding to the uncertainty and the free weighting matrices is discussed. In the contribution [2], it should be noted that functional $v_{a}$ does not vanish when $\mu \rightarrow 0$. The LMI condition is similar to the one here obtained. In the contribution [2], the free matrix $N 2$ is necessary in order to guarantee the negativity of the corresponding LMI for any upper-bound $\mu$. This fact is evidenced in element $\phi_{22}$. This gives sense to the introduction of the derivative quadratic term $-\dot{x}(t) Z \dot{x}(t)$ in the derivative of the proposed LKF (14). Additional benefits are obtained with the use of the past state $x(t-h)$ in the derivative of the LKF (14) as can be appreciated in element $\phi_{55}$.

For [4], firstly it should be noted that a little error is committed when the derivative of the nominal LKF along the perturbed system is calculated. Considering the nominal complete LKF $V_{n}$ (equation (19)) proposed in [4] and the system with uncertain delay (15), the correct derivative of the LKF along the perturbed system is

$$
\dot{V}_{n}\left(x_{t}\right)=-x^{T}(t) \tilde{W}_{0} x(t)-\dot{x}^{T}(t) \tilde{W}_{1} \dot{x}(t)+\tilde{\Delta},
$$


where $\tilde{\Delta}=-2\left[A_{1} \int_{t-h-\eta(t)}^{t-h} \dot{x}(s) d s\right]^{T}\left[\tilde{U}(0) x(t)+\tilde{W}_{1} \dot{x}(t)+\int_{-h}^{0} Q^{T}(h+\theta) A_{1} x(t+\theta) d \theta+\right.$ $\left.\frac{1}{2} \tilde{W}_{1} A_{1} \int_{t-h-\eta(t)}^{t-h} \dot{x}(s) d s\right]$. Here matrices $\tilde{U}(\cdot)$ and $Q(\cdot)$ are defined by (20a) and (29b) in [4], respectively. It is worth to mention that matrix $\tilde{U}(\theta)=\tilde{U}_{0}(\theta)+\tilde{U}_{1}(\theta)$ is defined in terms of a nominal delay Lyapunov matrix $\tilde{U}_{0}(\theta)$ and on a matrix satisfying an algebraic expression $\tilde{U}_{1}(\theta)=A_{0}^{T} X(\theta) A_{0}+A_{1}^{T} X(\theta+h) A_{0}+A_{0}^{T} X(\theta-h) A_{1}+A_{1}^{T} X(\theta) A_{1}$ and $X(\theta)=\int_{0}^{\infty} K^{T}(t) W_{1} K(t+\theta) d \theta \cdot K(\cdot)$ is the fundamental matrix for system (4). It should be pointed out that the key of the construction of the LKF proposed in [4] resides in the matrix $\tilde{U}_{0}(\theta)$. This is the principal difference with our contribution, the construction of the new LKF (14) just depends on a delay Lyapunov matrix $U\left(x_{t}, W\right)$ defined by (8)-(10) with $W$ satisfying (13). The cost for construct the new complete LKF (14) in a simple fashion is the introduction of cross terms in the derivative of the form $x^{T}(t) Z A_{0} \dot{x}(t)$. This will result in conservative estimation of upper-bound $\mu$ but marginal compared with the stability criteria if the same technique is applied to the LKF proposed in [4].

Example 1. Consider the system:

$$
\dot{x}(t)=\left(\begin{array}{cc}
0 & 1 \\
-1 & -2
\end{array}\right) x(t)+\left(\begin{array}{cc}
0 & 0 \\
-1 & 1
\end{array}\right) x(t-\tau(t)) .
$$

For $\tau(t)=h+\eta(t)$, where $h=1$ and $\eta(t)$ is a sign-varying piecewise continuous function satisfying $|\eta(t)| \leq \mu$.

With $W_{0}=1 I, W_{1}=W_{2}=0.0001 I$, by applying Theorem 1 we find that for $\mu=$ 0.11 the LMI involved is feasible. Hence, the system with uncertain non-small delay $\tau(t)=1+\eta(t)$ with $|\eta(t)| \leq 0.11$ is asymptotically stable. This upper-bound is the same as the one obtained with the LKF proposed in [4] with the correct derivative (19) and following the majorization procedure presented there. Following [2] with $W_{0}=1 I$, $W_{1}=W_{2}=0$ we find $\mu=0.049$.

With $W_{0}=1.5 I, W_{1}=0.0001 I, W_{2}=W_{1}$ we get from (12) that

$$
Z=\left[\begin{array}{ll}
1.6662 & 0.7424 \\
0.7424 & 0.8237
\end{array}\right]
$$

With $W=W_{0}+W_{1}+h_{1} W_{2}-A_{0}^{T} Z A_{0}+A_{1}^{T} Z A_{1}$, following a semi-analytic procedure we obtain

$$
U(0)=\left[\begin{array}{ll}
5.8238 & 1.1312 \\
1.1312 & 0.9164
\end{array}\right] \text { and } \mathscr{U}=\left[\begin{array}{cc}
28.2404 & 3.6132 \\
3.6132 & 0.9188
\end{array}\right]
$$

Then, by applying Theorem 1 we find that for $\mu=0.15$ the LMI involved is feasible. Hence, the system with uncertain non-small delay $\tau(t)=1+\eta(t)$ with $|\eta(t)| \leq 0.15$ is asymptotically stable. By considering the approach of free weighting matrices applied to the LKF presented in [4] with $\tilde{W}_{1}=\tilde{W}_{2}=I_{2 \times 2}$ and the additional functional (16) one may find a less conservative bound $\mu=0.17$. 


\section{Concluding Remarks}

In the present contribution a modification of complete LKFs is developed. The new complete LKF is used for the robust stability analysis of TDSs with uncertain fastvarying time delay arising from NCSs. It is evident that the computation of the delay Lyapunov matrices is crucial for a successful application of the quadratic functionals to the analysis of the uncertain time-delay systems. Given matrices $W_{0}, W_{1}$, and $W_{2}$, matrices $U(0)$ and $\mathscr{U}$ are used to obtain sufficient stability condition. A numerical example illustrates the design procedure.

\section{References}

1. Fridman, E.: A new Lyapunov technique for robust control of systems with uncertain nonsmall delay. Jornal of Mathematical Control and Information 23, 165-179 (2006)

2. Fridman, E., Niculescu, S.: On complete Lyapunov-Krasovskii functional techniques for uncertain systems with fast varying delays. Int. Journal of Robust and Nonliear Control 18, 364-374 (2008)

3. Fridman, E., Shaked, U.: A descriptor system approach to $H_{\infty}$ control of linear time-delay systems. IEEE Trans. Automat. Contr. 47(2), 253-270 (2002)

4. Fridman, E.: Stability of systems with uncertain delays: a new 'complete' LyapunovKrasovskii functional. IEEE Trans. Automat. Contr. 51, 885-890 (2006)

5. Gu, K., Kharitonov, V., Chen, J.: Stability of Time-Delay Systems. Boston (2003)

6. He, Y., Wang, Q., L.Xie, C.Lin: Further improvement of free weighting matrices technique for systems with time-varying delay. IEEE Trans. Automat. Contr. 52, 293-299 (2007)

7. Hespanha, J.P., Naghshtabrizi, P., Xu, Y.: A survey of recent results in networked control systems. PROCEEDINGS-IEEE 95(1), 138 (2007)

8. Kharitonov, V.L.: Lyapunov matrices: Existence and uniqueness issues. Automatica 46, $1725-1729$ (2010)

9. Kharitonov, V., E.Plischke: Lyapunov matrices for time-delay system. Systems \& Control Letters 55, 697-706 (2006)

10. Kharitonov, V., Niculescu, S.: On the stability of linear system with uncertain delay. IEEE Trans. Automat. Contr. 48(1), 127-132 (2003)

11. Kharitonov, V., Zhabko, A.: Lyapunov-Krasovskii approach to the robust stability analysis of time-delay system. Automatica 39, 15-20 (2003)

12. Meng, C., Wang, T., Chou, W., Luan, S., Zhang, Y., Tian, Z.: Remote surgery case: Robotassisted teleneurosurgery. In: IEEE Int. Conf. Robot. and Auto. (ICRA'04). vol. 1, pp. 819823 (2004)

13. Moon, Y., Park, P., Kwon, W., Lee, Y.: Delay-dependent robust stabilization of uncertain state-delayed systems. Int. Journal of Control 74(14), 1447-1455 (2001)

14. Ogren, P., Fiorelli, E., Leonard, N.: Coperative control of mobile sensor networks: Adaptive gradient climbing in a distributed environment. IEEE Trans. Automat. Contr. 49(8), 1292$1302(2004)$

15. Seiler, P., Sengupta, R.: Analysis of communication losses in vehicle control problems. In: Proc. 2001 Amer. Contr. Conf. vol. 2, pp. 1491-1496 (2001)

16. Seiler, P., Sengupta, R.: An $H_{\infty}$ approach to networked control. IEEE Trans. Automat. Contr. 50(3), 356-364 (2005)

17. de Souza, C., Li, X.: Delay-dependent robust $H_{\infty}$ control of uncertain linear state-delayed system. Automatica 35, 1313-1321 (1999)

18. Sun, J., Jiang, J.P.: Stability of uncertain networked control systems. Procedia Engineering 24, 551-557 (2011) 